\title{
P 032 ORGANISATIONAL FACTORS INFLUENCING UPTAKE OF A CARER SUPPORT NEEDS ASSESSMENT TOOL IN PALLIATIVE HOME CARE
}

L Austin, ${ }^{1}$ G Ewing, ${ }^{2} \mathrm{G}$ Grande'. ' $S$ School of Nursing, Midwifery and Social Work, University of Manchester; ${ }^{2}$ Centre for Family Research, University of Cambridge

10.1136/bmjspcare-2014-000838.35

Introduction The Carer Support Needs Assessment Tool (CSNAT) is a comprehensive, evidence-based tool for carer assessment and support. Successful implementation requires a change from existing practitioner led approaches to carer assessment to one which is facilitated by the practitioner, but led by the carer.

$\operatorname{Aim}(\mathrm{s})$ and method(s) To identify organisational factors which facilitate or hinder implementation of the CSNAT. The CSNAT was implemented in 6 palliative home care services. Qualitative data were collected via interviews (15), focus groups (2) and workshops (3), with practitioner 'champions' and service managers. All were audio-recorded, transcribed and analysed thematically.

Results Uptake of the CSNAT varied between services and was influenced by the receptivity of the context into which it was introduced. 'Organisational preparedness', with regard to existing administration systems, working patterns and competing demands, was a key factor. Additionally, facilitation to support implementation of the CSNAT was paramount; this included 'internal facilitation' from within the service (e.g. clear organisation steer) and 'external facilitation' from the research team (e.g. provision of training and on-going support).

Conclusion(s) The study identified two main factors which influence effective implementation of the CSNAT: a) receptivity of the organisational context and b) level of facilitation). These findings are in keeping with theory and practice models relating to sustainable implementation of practice developments. Further work is underway to explore how organisations can best be supported when implementing the CSNAT.

Funder NIHR Research for Patient Benefit. 